Barroso, ML, Moura, AMWA \& Pinto, NV. (2020). Correlation between general and abdominal obesity in diabetic and/or hypertensive active women. Research, Society and Development, 9(7):1-16, e179973679.

\title{
Correlação entre obesidade geral e abdominal em mulheres ativas diabéticas e/ou hipertensas
}

Correlation between general and abdominal obesity in diabetic and/or hypertensive active women

\section{Correlación entre obesidad general y abdominal en mujeres activas diabéticas y/o hipertensivas}

Recebido: 15/04/2020 | Revisado: 20/04/2020 | Aceito: 22/04/2020 | Publicado: 03/05/2020

Mateus Lemos Barroso

ORCID: https://orcid.org/0000-0002-3307-1636 Instituto Federal de Educação, Ciência e Tecnologia do Ceará, Brasil

E-mail: mateuslemosb@gmail.com Antônio Matheus Wilson Abreu Moura ORCID: https://orcid.org/0000-0001-7620-3304 Instituto Federal de Educação, Ciência e Tecnologia do Ceará, Brasil E-mail: matheuswilson.abreu@gmail.com Nilson Vieira Pinto

ORCID: https://orcid.org/0000-0001-6548-8586 Instituto Federal de Educação, Ciência e Tecnologia do Ceará, Brasil E-mail: nilsonvieira@ifce.edu.br

\section{Resumo}

Este estudo teve por objetivo investigar a correlação entre o índice de massa corporal e os indicadores antropométricos de obesidade abdominal em pacientes diabéticos e/ou hipertensos ativos assistidos pelo Núcleo de Apoio à Saúde da Família de Canindé, Ceará. Trata-se de uma pesquisa correlacional, transversal e de análise quantitativa. A amostra foi composta por 25 mulheres diabéticas e/ou hipertensas, ativas, com idade média de $63,64 \pm 10,25$ anos. Foram coletadas as medidas de peso, altura e circunferências da cintura (CC). O índice de massa corporal (IMC) foi calculado para identificar obesidade geral e a obesidade abdominal foi identificada com base nos indicadores CC, Relação Cintura/Estatura 
(RCE) e Índice de Conicidade (IC). Os resultados apontaram pacientes com sobrepeso (IMC $=28,3 \pm 4,0)$ e elevados índices antropométricos de obesidade abdominal $(\mathrm{CC}=100,1 \pm 8,7$; $\mathrm{RCE}=66,4 \pm 5,1 ; \mathrm{IC}=139,4 \pm 8,3)$. A CC $(\mathrm{p}<0,01 ; \mathrm{r}=0,826)$ e a $\operatorname{RCE}(\mathrm{p}<0,01 ; \mathrm{r}=0,730)$ se mostraram correlacionadas com o IMC nas pacientes hipertensas. Não foram identificadas correlações significativas entre os indicadores antropométricos de obesidade geral e abdominal na amostra de pacientes diabéticos e hipertensos-diabéticos. Em conclusão, identificou-se uma amostra de mulheres diabéticas e/ou hipertensas com sobrepeso e com risco elevado de desenvolver doenças associadas à obesidade. O IMC das pacientes hipertensas apresentou positiva correlação com a CC e com a RCE, mas não com o IC. Não houve correlações significativas nas pacientes diabéticas.

Palavras-chave: Índice de massa corporal; Antropometria; Obesidade; Diabetes; Hipertensão.

\begin{abstract}
This study aimed to investigate the correlation between body mass index and anthropometric indicators of abdominal obesity in diabetic and/or hypertensive active patients of the Health Family Support Nucleus in Caninde, Ceará. It is a correlational research, transverse and quantitative analysis. The sample consisted of 25 diabetic and/or hypertensive women, mean age $63.64 \pm 10.25$ years. The weight, height and waist circumference (WC) were collected. The body mass index (BMI) was calculated to identify general obesity and the abdominal obesity was identified based on WC indicators, waist/height ratio (WHR) and conicity index (CI). The results showed overweight patients $(\mathrm{BMI}=28.3 \pm 4.0)$ and high anthropometric indexes of abdominal obesity (WC $=100.1 \pm 8.7$; $\mathrm{WHR}=66.4 \pm 5.1 ; \mathrm{CI}=139.4 \pm 8.3)$. WC $(\mathrm{p}<0.01 ; \mathrm{r}=0.826)$ and WHR $(\mathrm{p}<0.01 ; \mathrm{r}=0.730)$ showed correlated with BMI in hypertensive patients. Significant correlations between anthropometric indicators of general and abdominal obesity of diabetic and hypertensive-diabetic patients were not identified. In conclusion, were identified a sample of diabetic and/or hypertensive women with overweight and high risk of developing diseases associated with obesity. BMI of hypertensive patients showed a positive correlation with the WC and the WHR, but not with the CI. There were no significant correlations in diabetic patients.
\end{abstract}

Keywords: Body mass index; Anthropometry; Obesity; Diabetes; Hypertension.

\title{
Resumen
}

Este estudio tuvo como objetivo investigar la correlación entre el índice de masa corporal y los indicadores antropométricos de obesidad abdominal en pacientes diabéticos y/o hipertensos asistidos por el Centro de salud familiar de Canindé, Ceará. Es un análisis correlacional, transversal y 
cuantitativo. La muestra consistió en 25 mujeres activas diabéticas y/o hipertensas, con una edad media de $63.64 \pm 10.25$ años. Se recogieron medidas de peso, altura y circunferencia de cintura (CC). El índice de masa corporal (IMC) se calculó para identificar la obesidad general y la obesidad abdominal se identificó en función de los indicadores CC, relación cintura/altura (RCA) e índice de conicidad (IC). Los resultados mostraron pacientes con sobrepeso (IMC $=28.3 \pm 4.0$ ) y altas tasas antropométricas de obesidad abdominal ( $\mathrm{CC}=100.1 \pm 8.7$; RCA $=66.4 \pm 5.1 ; \mathrm{IC}=139.4 \pm 8.3)$. CC (p $<0.01 ; \mathrm{r}=0.826)$ y RCA ( $\mathrm{p}<0.01 ; \mathrm{r}=0.730)$ mostraron correlación con el IMC en pacientes hipertensos. No se identificaron correlaciones significativas entre los indicadores antropométricos de obesidad general y abdominal en la muestra de pacientes diabéticos e hipertensos. En conclusión, se identificó una muestra de mujeres con sobrepeso y diabéticas con alto riesgo de desarrollar enfermedades asociadas con la obesidad. El IMC de pacientes hipertensos mostró una correlación positiva con CC y RCA, pero no con IC. No hubo correlaciones significativas en pacientes diabéticos.

Palabras clave: Índice de masa corporal; Antropometria; Obesidad; Diabetes; Hipertensión.

\section{Introdução}

A obesidade, compreendida como o acúmulo excessivo de gordura corporal em um indivíduo, é reconhecidamente um problema de saúde pública mundial capaz de elevar a probabilidade de desenvolver inúmeras doenças crônicas não transmissíveis, como diabetes e hipertensão arterial as quais favorecem o desenvolvimento de doenças cardiovasculares (Nascimento, Santos e Cardoso, 2014).

A antropometria vem sendo utilizada frequentemente como um instrumento de identificação dos riscos para desenvolver doenças cardiovasculares. Entre estes indicadores, o Índice de Massa Corporal (IMC) é o mais utilizado, o qual classifica a obesidade geral do indivíduo. Por sua vez, a obesidade abdominal, considerada um importante fator de risco para o desenvolvimento de doenças cardiovasculares, é identificada através de indicadores antropométricos como a Circunferência da Cintura (CC), a Relação Cintura/Estatura (RCE) e pelo índice de conicidade (IC). São medidas objetivas, simples, de baixo custo e fácil obtenção, útil em inquéritos populacionais, na prática clínica e em procedimentos preventivos e de acompanhamento em saúde (Vasques et al., 2010; Francischi et al., 2000).

A correlação entre o risco para desenvolver doenças cardiovasculares e os diversos indicadores antropométricos já tem sido estudada pela comunidade científica (Liberali et al., 2011; Farinea, Ricalde e Siviero, 2012; Oliveira et al., 2013) entretanto, a maioria dos estudos relacionados com adultos jovens e idosos apresentam relações restritas aos indicadores antropométricos de obesidade geral. Mesmo diante desta abordagem investigativa, ainda não 
se sabe qual a melhor correlação entre os indicadores antropométricos que apontem em potencial o risco de desenvolver doenças cardiovasculares, especialmente com pacientes obesos com diabetes e hipertensão arterial, os quais ainda representam uma população pouco investigada.

É percebido que a prevenção efetiva das doenças cardiovasculares é vislumbrada a partir da identificação do perfil de risco dos pacientes e populações. Desta forma, este estudo teve por objetivo identificar alguns indicadores antropométricos de obesidade e correlacionálos com as comorbidades diabetes e hipertensão arterial, uma vez que a identificação precoce dos riscos à obesidade nos serviços de Atenção Básica de Saúde fornece subsídios na avaliação antropométrica adequada e consequentemente na trajetória preventiva e terapêutica sobre o processo saúde-doença.

\section{Metodologia}

Trata-se de uma pesquisa correlacional, transversal e de análise quantitativa (Pereira et al., 2018), desenvolvida no Núcleo de Apoio a Saúde da Família (NASF) do município de Canindé, Ceará, composta por 25 mulheres, com idade entre 48 a 76 anos $(63,64 \pm 10,25)$ sendo, cinco diabéticas, 17 hipertensas e três com diabetes e hipertensão arterial, totalizando oito pacientes com diabetes mellitus e 20 com hipertensão arterial, diagnosticadas pela equipe de atenção básica a saúde, assistidas por esse núcleo de apoio.

Foram incluídas neste estudo todas as mulheres que estivessem ativas a um tempo mínimo de três meses, que concordassem em participar da pesquisa assinando o termo de consentimento livre e esclarecido, que fossem pacientes do NASF Canindé, portadoras de hipertensão arterial e/ou diabetes e que participassem assiduamente do programa de atividade física deste núcleo de apoio. Foram excluídas mulheres em acompanhamento temporário ou em trânsito; procedentes de outras regiões, por motivo de viagem, trabalho, passeio e outros; portadoras de transtornos mentais ou que tivessem alguma limitação física que pudesse interferir no seu nível de aptidão física.

Para a coleta de dados, inicialmente foram realizadas reuniões de caráter informativo e de reconhecimento junto a $5^{\text {a }}$. Coordenadoria Regional de Saúde e ao NASF de Canindé, a fim de identificar o atual serviço de assistência à saúde promovida aos pacientes diabéticos e hipertensos. Em sequência, foi realizada uma reunião in loco com todos os pacientes diabéticos e hipertensos assistidos pelo NASF Canindé, a fim de propor o estudo em questão e esclarecer todo o percurso metodológico. Neste encontro, ficou agendada uma nova visita no 
intuito de serem coletados os termos de consentimento livre e esclarecido àqueles que aceitarem participar da pesquisa e agendarmos as datas para aplicação dos testes paramétricos de avaliação da composição corporal.

No contexto dos testes paramétricos, o índice de massa corporal (IMC) foi utilizado como indicador antropométrico de obesidade geral. A circunferência da cintura (CC), a relação cintura/estatura (RCE) e o índice de Conicidade (IC) foram utilizados como indicadores antropométricos de obesidade abdominal. Todos os testes seguiram o padrão de aferição prescrito pela Organização Mundial da Saúde (2004).

O IMC foi obtido calculando o quociente da massa corporal (em $\mathrm{kg}$ ) pelo quadrado da altura em metros $\left(\mathrm{kg} / \mathrm{m}^{2}\right)$. Os dados relativos à massa corporal foram verificados com os participantes descalços e com roupas leves, utilizando-se uma balança portátil digital com capacidade para registrar $120 \mathrm{~kg}$ e uma precisão de $0,1 \mathrm{~kg}$, display automático acionado com o toque dos pés posicionado em chão reto (Balança Digital com estadiômetro; marca W721 Wiso). Para verificação da estatura foi realizada a medição com o avaliando descalço, estando com os pés juntos e paralelos, braços ao longo do corpo, posição ereta e a cabeça orientada no plano horizontal de Frankfurt (Machado e Abad, 2012).

Para avaliação da circunferência da cintura a fita métrica foi posicionada de forma horizontal no ponto médio entre a última costela e a crista ilíaca, estando o avaliado em pé com o abdômen relaxado e os braços estendidos ao lado do corpo. A relação entre a cintura e a estatura foi calculada pela razão entre a CC $(\mathrm{cm})$ e a altura $(\mathrm{cm})$ e para obter o IC, utilizouse a seguinte equação: $\mathrm{IC}=$ Circunferência de Cintura em metros $/(0,109$ x raiz quadrada da massa corporal em kg/estatura em metros).

Para análise estatística utilizou-se o software SPSS - Statistical Package for the Social Sciences - versão 16.0. Os dados se distribuíram de forma normal, conforme verificado por métodos gráficos e através das estatísticas de assimetria (skewness) e curtose (kurtosis). Para a análise descritiva, os parâmetros Índice de Massa Corporal (IMC), Circunferência da Cintura (CC), Relação Cintura-Estatura (RCE) e Índice de Conicidade (IC) foram representados pela média e desvio padrão. Estas foram comparadas entre os grupos Hipertensão e Diabetes pelo teste t de Student. A correlação entre o IMC, CC, RCE e IC foi analisada pelo coeficiente de correlação de Pearson para a amostra geral e estratificada conforme o perfil de comorbidades.

Este estudo seguiu-se dentro das normas que regulamentam a pesquisa em seres humanos, do Conselho Nacional de Saúde - Ministério da Saúde, Resolução No 466/2012 e encontra-se aprovado pelo CEP/IFCE sob o Parecer $n^{\circ} 432.503 / 2013$. 


\section{Resultados e Discussão}

$\mathrm{Na}$ análise do indicador de obesidade geral da amostra total, o IMC apontou um índice médio de 28,3( $\pm 4,0)$, categorizando este grupo com sobrepeso. Na avaliação dos indicadores de obesidade abdominal da amostra total, a CC apontou valor médio de 100,1( $\pm 8,7)$, a RCE de $66,4( \pm 5,1)$ e IC de 139,4( $( \pm 8,3)$ classificando esta amostra com "risco muito aumentado" para aquisição de morbidades relacionadas ao excesso de peso (Tabela 1).

$\mathrm{Na}$ avaliação do indicador de obesidade geral nas pacientes hipertensas, foi obtido um índice médio de 28,6( $\pm 4,1)$, caracterizando o grupo com sobrepeso. Ao analisarmos os indicadores de obesidade abdominal, a CC apresentou valor médio de $101,1( \pm 8,9)$, o que representa um "risco muito aumentado" para morbidades associadas à obesidade. Estes dados são enfatizados ao se identificar os valores de $66,9( \pm 4,6)$ para a RCE e $140,7( \pm 8,1)$ para o IC (Tabela 1).

Tabela 1. Valores de IMC, CC, RCE e IC na amostra geral e segundo as comorbidades Hipertensão e Diabetes (média e desvio padrão).

\begin{tabular}{cccccc} 
Parâmetro & $\begin{array}{c}\text { Não hipertenso } \\
(\mathbf{n = 5})\end{array}$ & $\begin{array}{c}\text { Hipertenso } \\
(\mathbf{n = 2 0})\end{array}$ & $\begin{array}{c}\text { Não diabético } \\
(\mathbf{n = 1 7})\end{array}$ & $\begin{array}{c}\text { Diabético } \\
(\mathbf{n = 8})\end{array}$ & $\begin{array}{c}\text { Total } \\
(\mathbf{n = 2 5})\end{array}$ \\
\hline IMC & $27,3 \pm 3,8$ & $28,6 \pm 4,1$ & $29,1 \pm 4,1$ & $26,7 \pm 3,4$ & $28,3 \pm 4,0$ \\
CC & $96,0 \pm 7,3$ & $101,1 \pm 8,9$ & $102,2 \pm 9,1$ & $95,5 \pm 5,8$ & $100,1 \pm 8,7$ \\
RCE & $64,3 \pm 7,0$ & $66,9 \pm 4,6$ & $67,7 \pm 4,4$ & $63,7 \pm 5,7$ & $66,4 \pm 5,1$ \\
IC & $133,9 \pm 7,1$ & $140,7 \pm 8,1$ & $141,1 \pm 8,7$ & $135,7 \pm 6,3$ & $139,4 \pm 8,3$ \\
\hline
\end{tabular}

Fonte: Elaborado pelo autor

A intima relação entre a obesidade e a hipertensão arterial vem amplamente discutida pela comunidade científica. Ferreira \& Zanella (2000) comentam que indivíduos obesos possuem até oito vezes mais chances de serem acometidos pela hipertensão do que os com peso normal.

Nascente et al. (2009) investigaram a interferência da distribuição da gordura corporal sobre a prevalência de hipertensão e outras patologias, enfatizando que indivíduos com obesidade detém maior probabilidade de apresentar problemas cardiovasculares do que indivíduos com o peso adequado. Este autor comenta ainda, uma constante relação encontrada entre a prevalência acentuada de obesos hipertensos com a hiperinsulinemia. Tal relação se baseia no fato de que a quantidade elevada de insulina gera a ativação do sistema nervoso 
simpático, que absorve maiores quantidades de sódio, a partir disto eleva-se a resistência vascular periférica e a pressão arterial.

Carneiro et al. (2003) realizaram um estudo relacionando a composição corporal e a hipertensão arterial de 499 indivíduos, dos quais 432 eram mulheres e detectaram a prevalência de hipertensão em 43,8\% da amostra. Esta prevalência era de $23 \%$ nos indivíduos com sobrepeso e de $67,1 \%$ nos pacientes com obesidade grau $3(\mathrm{p}<0.05)$.

Moraes, Checchio \& Freitas (2015) identificaram uma prevalência de 44,5\% de mulheres hipertensas em uma amostra de 2.471 adultos, onde os indicadores antropométricos de obesidade abdominal mostraram-se associados a doença crônica.

Andrade et al. (2014) verificaram a prevalência de hipertensão, fatores de risco e complicações em 220 idosos e identificaram que 51,4\% (n=113) da amostra analisada era composta de hipertensos, sendo que 63,7\% $(n=72)$ eram mulheres, 64,6\% $(n=73)$ eram inativos, 52,2\% $(\mathrm{n}=59)$ foram caracterizados com sobrepeso e 53,1\% ( $\mathrm{n}=60)$ com obesidade abdominal. Desta amostra 17,7\% $(n=20)$ apresentavam comorbidades e complicações e 29,2\% $(n=33)$ eram diabéticos.

$\mathrm{Na}$ avaliação do indicador de obesidade geral nas pacientes diabéticas, o IMC apontou um valor médio de 26,7( $\pm 3,4)$ classificando-as com sobrepeso. Nos parâmetros de obesidade abdominal, a CC apresentou valor médio de 95,5( $\pm 5,8)$, evidenciando o "risco muito aumentado" para o desenvolvimento de morbidades relacionadas à obesidade. Por sua vez, os valores médios apontados pela RCE $63,7( \pm 5,7)$ e pelo IC $135,7( \pm 6,3)$ enfatizam o alto risco de comorbidades associadas ao excesso ponderal (Tabela 1).

A relação entre a obesidade e o diabetes, assim como a hipertensão arterial, também tem sido explorada. Em um estudo multicêntrico realizado por Picon et al. (2007) identificou mulheres diabéticas com sobrepeso, apresentando IMC médio de 28,1 $( \pm 4,7)$ e valores médios da CC de 96,9( \pm 12$)$, representando, assim como encontrado em nossa amostra, risco muito aumentado para o desenvolvimento de comorbidades relacionadas ao excesso de peso.

Gomes et al. (2006) analisaram a prevalência de sobrepeso e obesidade em 2.519 pacientes diabéticos, com idade de $58,8( \pm 11,6)$ anos, e perceberam que pacientes com peso normal apresentaram menor índice de controle glicêmico e, que aqueles em tratamento com associação de duas ou mais drogas orais e associação de insulina e droga oral apresentaram maior IMC do que aqueles em tratamento com dieta, hipoglicemiante oral e insulina.

Faustino et al. (2011) investigaram a prevalência de obesidade em 108 pacientes diabéticos, dos quais 78,6\% eram mulheres. Os resultados deste estudo apontaram uma 45,6\% 
das mulheres avaliadas eram obesas e 94,2\% da amostra geral apresentaram "risco muito aumentado" para o desenvolvimento de doenças associadas à obesidade.

Em adição, Siqueira et al. (2015), em um estudo populacional com 1.161 indivíduos adultos acima de 40 anos, identificou que a prevalência da obesidade abdominal em mulheres era mais que o dobro em relação aos homens, apontando a hipertensão arterial e o diabetes como variáveis associadas a estes índices.

Estes estudos citados acima, evidenciam a elevada prevalência de hipertensão arterial e diabetes no sexo feminino, destacando o sedentarismo, o sobrepeso e a obesidade abdominal como fatores de riscos diretamente correlacionados, uma relação que corrobora com os resultados identificados em nossa amostra.

A caracterização de uma amostra de pacientes com sobrepeso, independente da comorbidade (hipertensão e/ou diabetes) e, que a partir dos indicadores de obesidade abdominal, sinalizam para o desenvolvimento de outras possíveis comorbidades associadas à obesidade ou mesmo ao agravo destas, faz com que as estratégias preventivas e terapêuticas adotadas pelo NASF de Canindé sejam refletidas e fortalecidas quanto aos seus procedimentos e monitoramento.

Torna-se preocupante identificar valores de obesidade geral e abdominal tão expressivos em um grupo de mulheres ativas, que participam de um programa regular de atividade física orientada e que mesmo acompanhadas, ainda não demonstram alterações positivas na composição corporal que possam auxiliar na terapêutica da doença.

Vale ressaltar que aspectos nutricionais, socioeconômicos e demográficos não foram pontos de análise e correlação neste estudo, sendo parâmetros que possam inferir na composição corporal desta amostra. Esses aspectos serão avaliados em estudos futuros.

A prevenção e promoção da saúde através do estímulo a mudança no estilo de vida dessas pacientes, quer seja pela adoção de hábitos alimentares saudáveis, quer seja pela prática regular de atividade física, poderá fortalecer o acompanhamento e a terapêutica necessária no controle da obesidade e de suas doenças associadas.

Em uma análise correlacional da amostra geral (Tabela 2), a CC (p<0,01; $r=0,795)$ e a RCE $(\mathrm{p}<0,01 ; \mathrm{r}=0,732)$ se mostraram correlacionadas com o IMC, tendo o IC apresentado correlação apenas com a CC ( $\mathrm{p}<0,01 ; \mathrm{r}=0,560)$. Estes dados correlacionais estão ilustrados no gráfico 1. 
Research, Society and Development, v. 9, n. 7, e179973679, 2020

(CC BY 4.0) | ISSN 2525-3409 | DOI: http://dx.doi.org/10.33448/rsd-v9i7.3679

Tabela 2. Correlação entre os parâmetros IMC, CC, RCE e IC para a amostra geral (n=25)

\begin{tabular}{ccccc}
\hline & IMC & CC & RCE & IC \\
\hline IMC & - & $0,795^{* *}$ & $0,732^{* *}$ & $-0,006$ \\
CC & $0,795^{* *}$ & - & $0,566^{* *}$ & $0,560^{* *}$ \\
RCE & $0,732^{* *}$ & $0,566^{* *}$ & - & 0,072 \\
IC & $-0,006$ & $0,560^{* *}$ & 0,072 & - \\
\hline
\end{tabular}

Os valores apresentados correspondem ao coeficiente de correlação de Pearson; ** $\mathrm{p}<0.01$

Fonte: Elaborado pelo autor

Gráfico 1. Correlação e intervalo de confiança de 95\% para os parâmetros IMC, CC, RCE e IC na amostra geral $(n=25)$.

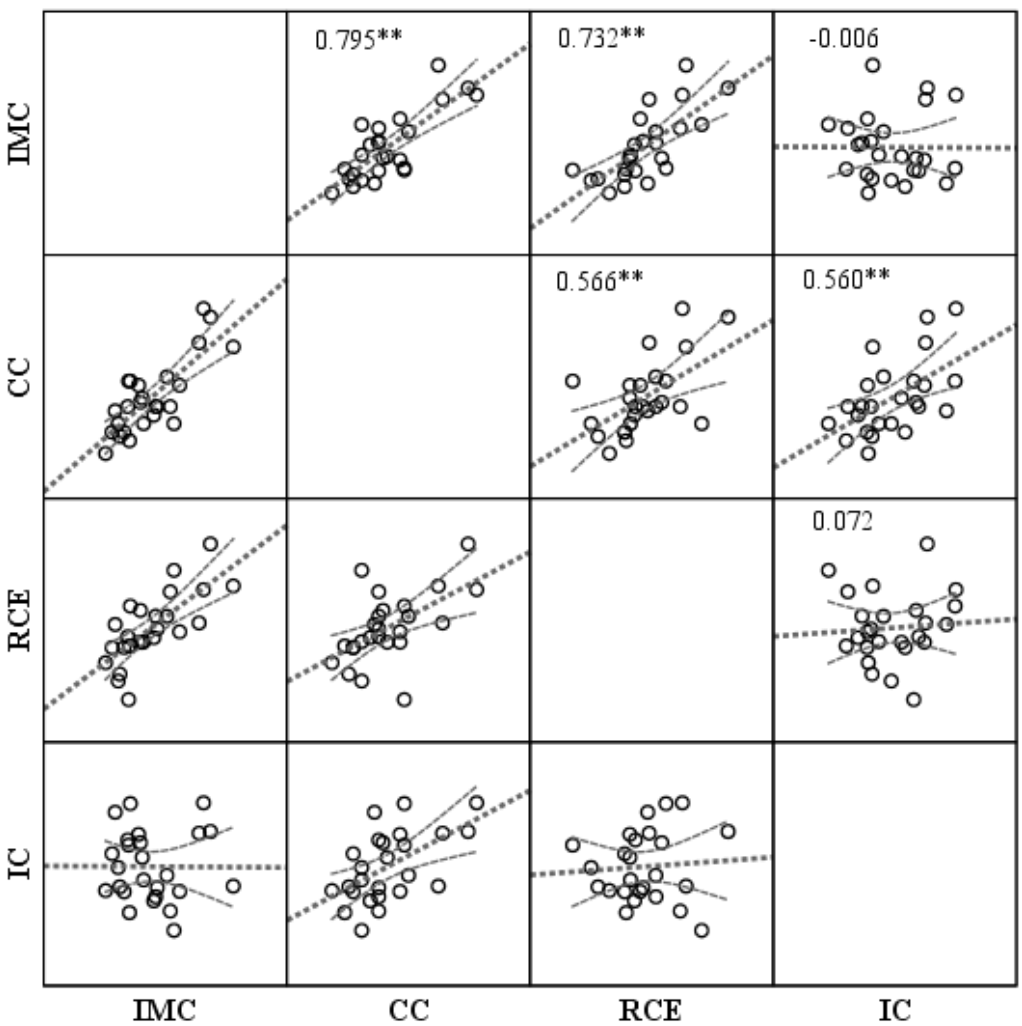

Em uma análise correlacional por comorbidade, a $\mathrm{CC}(\mathrm{p}<0,01 ; \mathrm{r}=0,840)$ e a $\mathrm{RCE}$ $(\mathrm{p}<0,01 ; \mathrm{r}=0,755)$ se mostraram correlacionadas com o IMC nas pacientes hipertensas e hipertensas-diabéticas (Tabela 3), tendo o IC apresentado correlação apenas com a CC $(\mathrm{p}<0,05 ; \mathrm{r}=-0,529)$. Estes resultados são semelhantes aos encontrados na correlação dos indicadores antropométricos da amostra geral. 
Tabela 3. Correlação entre os parâmetros IMC, CC, RCE e IC segundo a comorbidade Hipertensão.

\begin{tabular}{ccccc}
\hline \multicolumn{1}{c}{ Hipertensão } & & & \\
Não (n=5) & IMC & CC & RCE & IC \\
\hline IMC & & & 0,694 & $-0,480$ \\
$\mathbf{C C}$ & $0,840^{* *}$ & 0,514 & $-0,233$ & 0,500 \\
$\mathbf{R C E}$ & $0,755^{* *}$ & $0,767^{* *}$ & - & $-0,954^{*}$ \\
$\mathbf{I C}$ & 0,020 & $0,529^{*}$ & 0,265 & - \\
\hline
\end{tabular}

Os valores apresentados correspondem ao coeficiente de correlação de Pearson.

$* \mathrm{p}<0.05 ; * * \mathrm{p}<0.01$

Fonte: Elaborado pelo autor

Alguns estudos têm relacionado os indicadores antropométricos de obesidade com fatores de risco para o desenvolvimento de doenças cardiovasculares. Chuang et al. (2012) mostraram uma relação direta entre o desenvolvimento de doenças cardiovasculares e síndrome metabólica com o IMC e CC.

Rezende et al. (2006) demonstraram uma relação diretamente proporcional entre o IMC e CC e o agravo de fatores de risco para o desenvolvimento de doenças cardiovasculares. O estudo de Peixoto et al. (2006) caracterizou uma amostra com a CC em nível $1(\geq 80 \mathrm{~cm})$ e o IMC $\geq 25 \mathrm{~kg} / \mathrm{m}^{2}$, denotando pontos de corte com efetiva capacidade preditiva de hipertensão arterial.

Estes estudos corroboram com os resultados encontrados nas pacientes hipertensas e reafirmam a utilização do IMC, CC e RCE como indicadores antropométricos valiosos na predição e no monitoramento da obesidade e da hipertensão arterial sistêmica. Por sua vez, o IC parece não ser um bom indicador antropométrico nesta correlação, visto os resultados apontados em nosso estudo e fortalecido pelas investigações dos autores citados anteriormente.

Em contradição aos resultados encontrados no grupo de pacientes hipertensas, a avaliação correlacional dos indicadores antropométricos das pacientes diabéticas e diabéticashipertensas (Tabela 4), não apresentou correlação estatisticamente significativa dos indicadores de obesidade abdominal utilizados neste estudo com o IMC. Em adição, o IC apresentou correlação inversa com o RCE $(\mathrm{p}<0,01 ; \mathrm{r}=-0,860)$. Este resultado pode estar 
relacionado ao baixo número de pacientes diabéticos na amostra avaliada, que não promovem expressiva correlação quando analisados estatisticamente.

Tabela 4. Correlação entre os parâmetros IMC, CC, RCE e IC segundo a comorbidade Diabetes.

\begin{tabular}{ccccc}
\hline \multicolumn{1}{c}{ Diabetes } & CC & RCE & IC \\
Sim (n=8) & IMC $(\mathbf{n = 1 7 )}$ & & & \\
\hline IMC & & $0,826^{* *}$ & $0,730^{* *}$ & 0,024 \\
$\mathbf{C C}$ & 0,574 & - & $0,758^{* *}$ & $0,553^{*}$ \\
$\mathbf{R C E}$ & 0,694 & $-0,136$ & - & 0,280 \\
IC & $-0,594$ & 0,265 & $-0,860^{* *}$ & - \\
\hline
\end{tabular}

Os valores apresentados correspondem ao coeficiente de correlação de Pearson.

$* \mathrm{p}<0.05 ; * * \mathrm{p}<0.01$

Fonte: Elaborado pelo autor

Alguns estudos têm descrito a correlação entre os parâmetros de controle clínico e metabólico do diabetes e os indicadores antropométricos de obesidade central. Moraes et al. (2003) investigou pacientes diabéticos de ambos os sexos e distintas faixas etárias e alertaram para a tendência mundial do aumento de peso e de suas consequências clínicas observando, em especial, o agravo da pressão arterial dos diabéticos correlacionados aos elevados índices de obesidade abdominal.

No estudo correlacional de Machado et al. (2012) identificou-se que a CC e o RCE se correlacionaram positivamente com o IMC em 130 pacientes diabéticos, apesar de não apresentar correlação com o IC. Estes resultados, embora sejam contraditórios aos nossos achados nas pacientes diabéticas, se assemelham aos encontrados nas pacientes hipertensas, reconhecendo a CC e o RCE como bons indicadores de obesidade abdominal e correlacionados a obesidade geral.

Acredita-se que o resultado obtido nas pacientes diabéticas e hipertensas-diabéticas esteja relacionado ao baixo quantitativo amostral que quando analisado estatisticamente, não apresentaram significância. Uma relação passível de ocorrer em estudos clínicos e que denota a necessidade de estudos multicêntricos na tentativa de se ampliar o olhar desta correlação em pacientes diabéticos, diabéticos-hipertensos e com outras comorbidades. 
Todavia, pôde-se perceber uma positiva correlação entre o IMC, CC e RCE em pacientes hipertensos, tendo nesta análise a $\mathrm{CC}$ o destaque na predição da obesidade abdominal e na correlação com a obesidade geral.

Estes resultados apontam para a necessidade de reflexão quanto aos procedimentos de acompanhamento e monitoramento da obesidade promovidos pelo NASF em Canindé e destacam a importância de ações educativas e transformadoras do estilo de vida destes pacientes.

Neste contexto, a inserção do profissional de Educação Física nos serviços de atendimento básico a saúde se faz de suma importância, no intuito de promover as orientações necessárias na prática de exercícios físicos, ferramenta fundamental na melhora da qualidade de vida e na redução dos fatores de risco relacionados à obesidade. Infelizmente, ainda há a necessidade de reconhecimento e inserção deste profissional na estratégia de Saúde da Família. Martinez; Silva; Silva (2014) apontam que há um profissional de Educação Física para cada 100.000 habitantes no Brasil, uma baixa estimativa frente aos demais profissionais da saúde e a necessidade real deste profissional junto à população.

Embora existam inúmeras produções científicas que abordem a obesidade e seus indicadores antropométricos, entre as limitações deste estudo, destaca-se o exíguo referencial teórico capaz de fortalecer a discussão das correlações destes indicadores antropométricos de obesidade em pacientes diabéticos e/ou hipertensos, o que valida este trabalho e promove a necessidade de novos estudos em diferentes populações, a fim de se ampliar o entendimento destas relações nexo-causais.

\section{Conclusão}

Este estudo identificou uma amostra de pacientes diabéticas e/ou hipertensas ativas classificadas com sobrepeso e com risco elevado de desenvolver doenças associadas à obesidade. O índice de massa corporal apresentou positiva correlação com a circunferência da cintura e com a razão cintura/estatura, mas não com o índice de conicidade, nas pacientes hipertensas. Não foram identificadas correlações significativas entre os indicadores antropométricos de obesidade geral e abdominal na amostra de pacientes diabéticos e hipertensos-diabéticos.

Estes resultados conduzem a reflexão quanto as estratégias e procedimentos atualmente adotados nos serviços de Atenção Básica de Saúde no controle da obesidade e fortalecem o uso do índice de massa corporal, da circunferência da cintura e da razão 
cintura/estatura como preditores de obesidade geral e abdominal, podendo ainda serem correlacionados em pacientes com hipertensão arterial sistêmica.

Como estratégias investigativas futuras, sugere-se estudos multicêntricos que possam ampliar as possibilidades de correlação entre os indicadores de obesidade geral e abdominal em diferentes gêneros, amostras populacionais e comorbidades associadas.

\section{Referências}

Andrade, A. O., de Aguiar, M. I. F., de Almeida, P. C., Chaves, E. S., Araujo, N. V. S. S., \& de Freitas Neto, J. B. (2014). Prevalência da hipertensão arterial e fatores associados em idosos. Revista Brasileira em Promoção da Saúde, 27(3), 303-311.

Carneiro, G., Faria, A. N., Ribeiro Filho, F. F., Guimarães, A., Lerário, D., Ferreira, S. R., \& Zanella, M. T. (2003). Influência da distribuição da gordura corporal sobre a prevalência de hipertensão arterial e outros fatores de risco cardiovascular em indivíduos obesos. Revista da Associação Médica Brasileira, 49(3), 306-311.

Chuang, H. H., Li, W. C., Sheu, B. F., Liao, S. C., Chen, J. Y., Chang, K. C., \& Tsai, Y. W. (2012). Correlation between body composition and risk factors for cardiovascular disease and metabolic syndrome. Biofactors, 38(4), 284-291.

Liberali, R., Ribeiro, M. R., Aguiar, R. M., Cavalcanti, R. L., Batista, V. B., Liberali, R., \& Coutinho, V. F. (2011). Perfil nutricional de adultos e idosos atendidos na rede municipal de saúde de Manaus e sua associação a doenças crônico-degenerativas. Revista Brasileira de Ciências do Envelhecimento Humano, 8(1).

Farinea, N., Ricalde, S. R., \& Siviero, J. (2010). Perfil nutricional e antropométrico de idosos participantes de um grupo de ginástica no município de Antônio Prado-RS. Revista Brasileira de Ciências do Envelhecimento Humano, 7(3).

Faustino, E. B., de Azevedo, E. B., de Castro Silva, P. M., dos Santos Sales, S., \& de Sousa Barros, W. P. (2011). Diabetes melittus: busca ativa em portadores de obesidade. Cogitare Enfermagem, 16(1), 110-115. 
Ferreira, S. R. G., \& Zanella, M. T. (2000). Epidemiologia da hipertensão arterial associada à obesidade. Rev bras hipertens, 7(2), 128-35.

Francischi, R. P. P. D., Pereira, L. O., Freitas, C. S., Klopfer, M., Santos, R. C., Vieira, P., \& Lancha Júnior, A. H. (2000). Obesidade: atualização sobre sua etiologia, morbidade e tratamento. Revista de Nutrição, 13(1), 17-28.

Gomes, M. D. B., Giannella Neto, D., Mendonça, E. D., Tambascia, M. A., Fonseca, R. M., Réa, R. R., ... \& Cavalcanti, S. (2006). Prevalência de sobrepeso e obesidade em pacientes com diabetes mellitus do tipo 2 no Brasil: estudo multicêntrico nacional. Arquivos Brasileiros de Endocrinologia \& Metabologia, 50(1), 136-144.

Machado, A. F., \& Abad, C. C. C. (2012). Manual de avaliação física.

Machado, S. P., Rodrigues, D. G. C., Viana, K. D. A. L., \& de Carvalho Sampaio, H. A. (2012). Correlação entre o índice de massa corporal e indicadores antropométricos de obesidade abdominal em portadores de diabetes mellitus tipo 2. Revista Brasileira em Promoção da Saúde, 25(4), 512-520.

Martinez, J. F. N., Silva, A. M., \& Silva, M. S. (2014). As diretrizes do NASF e a presença do profissional em Educação Física. Motrivivência, 26(42), 222-237.

Moraes, S. A. D., Checchio, M. V., \& Freitas, I. C. M. D. (2015). O efeito independente da obesidade central sobre a hipertensão arterial em adultos residentes em Ribeirão Preto, SP, 2007. Projeto EPIDCV. Revista Brasileira de Epidemiologia, 18(1), 157-173.

Moraes, C. M., Portella, R. B., Pinheiro, V. S., Oliveira, M. M. S., Fuks, A. G., Cunha, E. F., et al. (2003). Prevalence of overweight and obesity in type 1 diabetic patients. Arq. Bras. Endocrinol. Metab., 47(6), 677-83.

Nascente, F. M. N., Jardim, P. C. B. V., Peixoto, M. D. R. G., Monego, E. T., Barroso, W. K. S., Moreira, H. G., ... \& Scala, L. N. (2009). Hipertensão arterial e sua associação com índices antropométricos em adultos de uma cidade de pequeno porte do interior do Brasil. Revista da Associação Médica Brasileira, 55(6), 716-722. 
Nascimento, R. G., Santos, Z. L., \& Cardoso, R. O. (2014). Desempenho de indicadores de obesidade abdominal e risco cardiovascular de idosos atendidos na rede básica de saúde do município de Belém-PA. Revista Brasileira de Ciências do Envelhecimento Humano, 11(2), 119-30.

Oliveira, L. M. F. T. D., Ritti-Dias, R. M., Amorim, R. A., Oliveira, S. F. M. D., Lucena Filho, A., \& Guimarães, F. J. D. S. P. (2013). Associação entre obesidade geral e abdominal com a hipertensão arterial em idosas ativas. Revista da Educação Física/UEM, 24(4), 659668.

OMS. Obesidade: prevenindo e controlando a epidemia global. Relatório da consultoria da OMS. Tradução: Andréa Favano; revisão científica: Sérgio Setsuo Maeda. São Paulo: Roca; 2004.

Peixoto, M. D. R. G., Benício, M. H. D. A., Latorre, M. D. R. D. D., \& Jardim, P. C. B. V. (2006). Circunferência da cintura e índice de massa corporal como preditores da hipertensão arterial. Arquivos Brasileiros de Cardiologia, 87(4), 462-470.

Pereira, A.S. et al. (2018). Metodologia da pesquisa científica. [e-book]. Santa Maria. Ed. UAB/NTE/UFSM. Disponível em: <

https://repositorio.ufsm.br/bitstream/handle/1/15824/Lic_Computacao_MetodologiaPesquisa-Cientifica.pdf?sequence=1> Acesso em 07 abr 2020

Picon, P. X., Leitão, C. B., Gerchman, F., Azevedo, M. J. D., Silveiro, S. P., Gross, J. L., \& Canani, L. H. (2007). Medida da cintura e razão cintura/quadril e identificação de situações de risco cardiovascular: estudo multicêntrico em pacientes com diabetes melito tipo 2. Arquivos Brasileiros de Endocrinologia \& Metabologia, 51(3), 443-449.

Rezende, F. A. C., Rosado, L. E. F. P. L., Ribeiro, R. D. C. L., Vidigal, F. D. C., Vasques, A. C. J., Bonard, I. S., \& Carvalho, C. R. D. (2006). Índice de massa corporal e circunferência abdominal: associação com fatores de risco cardiovascular. Arquivos Brasileiros de Cardiologia, 87(6), 728-734. 
Siqueira, D. G. B., Souza, R. K. T. D., Mesas, A. E., Santos, H. G. D., \& Bortoletto, M. S. S. (2015). Diferenças entre sexos nos determinantes da obesidade abdominal em adultos de 40 anos ou mais: estudo de base populacional. Revista de nutrição, 28(5), 485-496.

Vasques, A. C. J., Priore, S. E., Rosado, L. E. F. P. D., \& Franceschini, S. D. C. C. (2010). Utilização de medidas antropométricas para a avaliação do acúmulo de gordura visceral. Revista de nutrição, 23(1), 107-118.

Porcentagem de contribuição de cada autor no manuscrito

Mateus Lemos Barroso - 35\%

Antônio Matheus Wilson Abreu Moura - 30\%

Nilson Vieira Pinto - 35\% 